\title{
Rapid Freezing of a Water Droplet on an Aligned Si Nanorod Array Substrate
}

\author{
J.-G. Fan ${ }^{1}$ and Y.-P. Zhao ${ }^{2}$ \\ ${ }^{1}$ Center for Advanced Ultrastructural Research \\ ${ }^{2}$ Department of Physics and Astronomy \\ The University of Georgia, Athens, GA 30602
}

\begin{abstract}
When a water droplet is dried on a vertically aligned Si nanorod array surface, the nanorods are bundled together. To understand how bundles are formed, a water droplet is frozen rapidly on a Si nanorod array surface and in-situ cleaved for cryo-SEM. The nanorods in the precursor film form similar bundles as those dried in air. But the nanorods under the apparent frozen water droplet are only slightly deformed. We propose that the bundling of nanorods is caused by nonuniform water-nanorod interaction, which could happen either during the water spreading or drying process. Therefore, controlling the liquid-nanostructure interaction could minimize the bundling. In addition, the rapid freezing process does not preserve the water inside the nanochannels, and almost all the water forms ice on top of the nanorod surface, either as a planar interface or as particles, depending on the locations. The separated ice-nanorod interface will have potential applications in chemical separation and crystal growth.
\end{abstract}
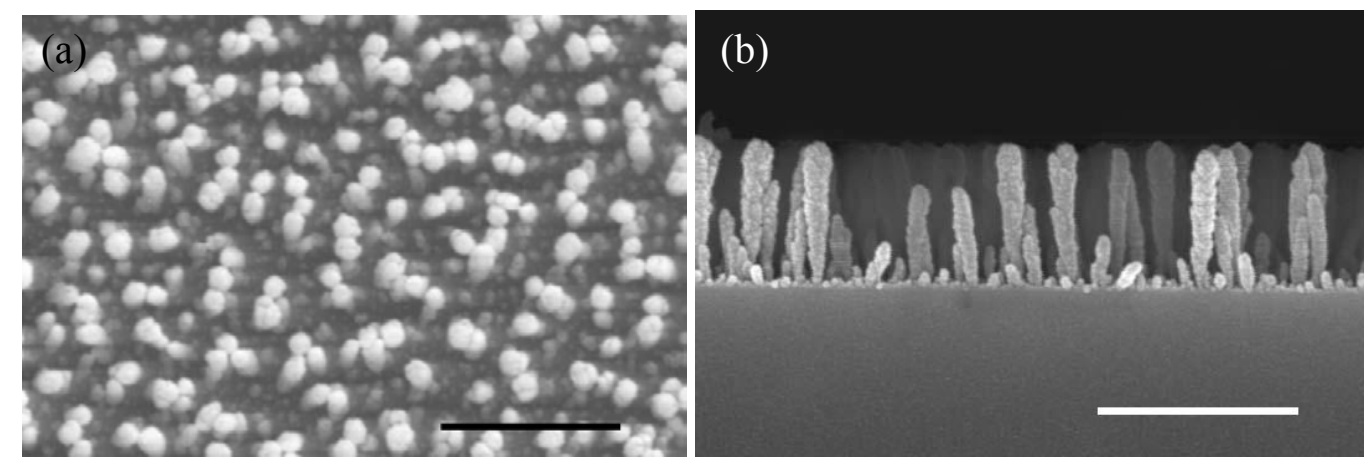

Fig. 1 SEM images of as-deposited Si nanorod arrays: (a) top-view and (b) cross-section. The scale bars are $1 \mu \mathrm{m}$.

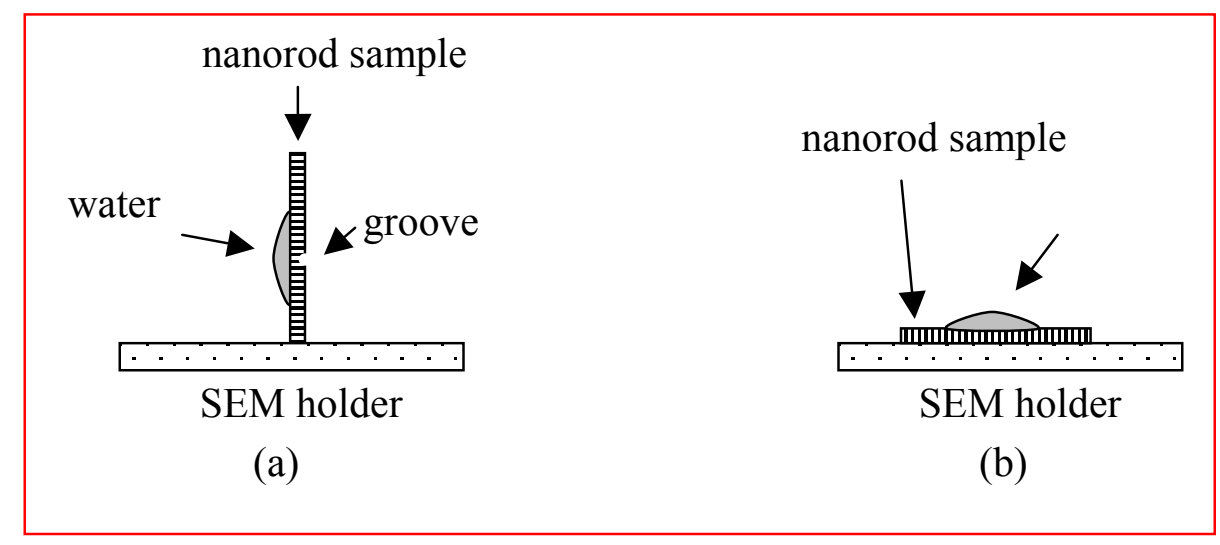

Fig. 2 The rapid freezing experiment for (a) cross-section SEM imaging, and (b) top-view SEM imaging. 

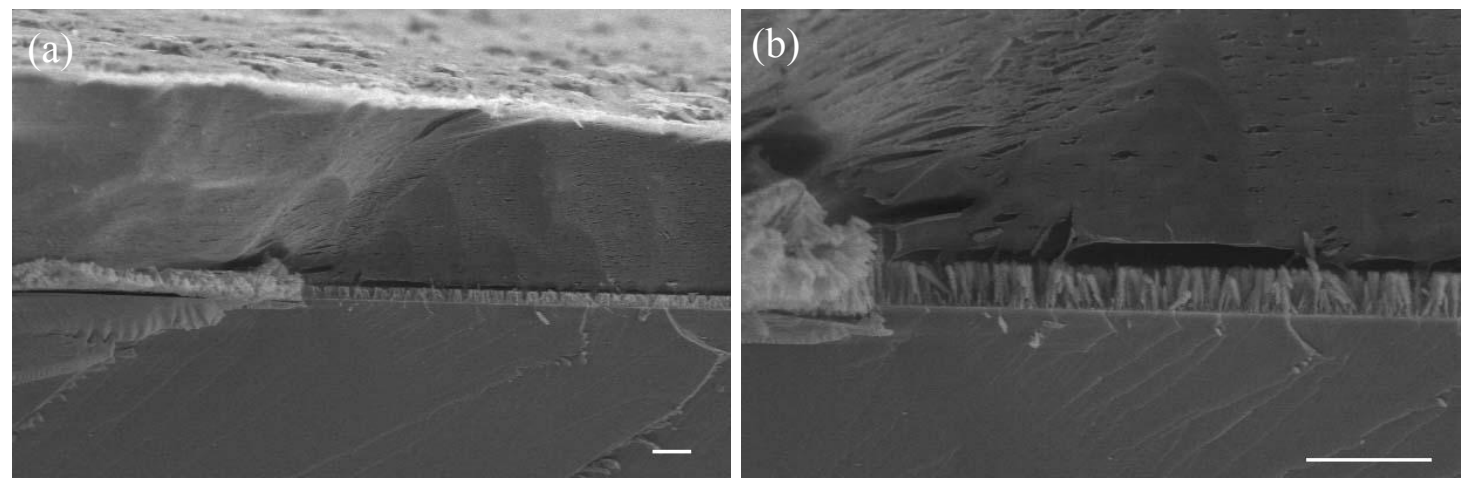

Fig. 3 Cross-section SEM images of the ice-nanorod interface with different magnifications. The scale bars are $2 \mu \mathrm{m}$.

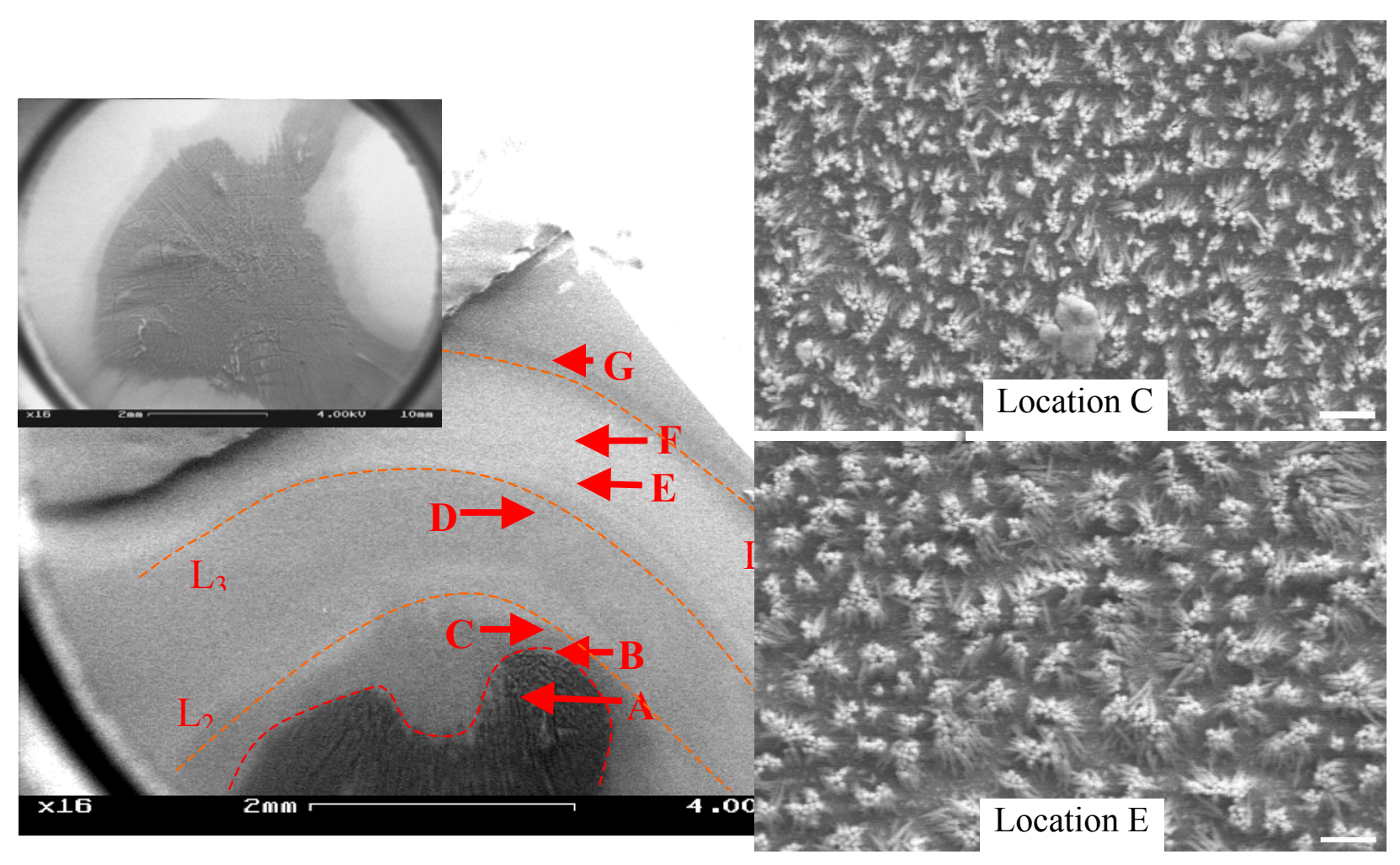

Fig. 4 Top-view SEM images of a frozen water droplet (left) and representative zoom-in images at specific locations (right: scale bars are $1 \mu \mathrm{m}$ ): the nanorod patterns are location dependent and are closely related to the dynamic water spreading process 